\title{
An efficient method to identify virus-specific TCRs for TCR-T cell immunotherapy against virus-associated malignancies
}

\author{
Lei Chen ${ }^{1+}$, Lianhua Dong ${ }^{1 \dagger}$, Yipeng Ma', Juntao Wang ${ }^{1}$, Dongjuan Qiao ${ }^{1}$, Geng Tian ${ }^{2}$ and Mingjun Wang ${ }^{1 *}$
}

\begin{abstract}
Adoptive transfer of T cells genetically engineered with a T cell receptor (TCR) is a promising cancer treatment modality that requires the identification of TCRs with good characteristics. Most T cell cloning methods involve a stringent singularization process, which necessitates either tedious hands-on operations or high cost. We present an efficient and nonstringent cloning approach based on existing techniques. We hypothesize that after elimination of most nonspecific T cells, a clonotype with high quality could outcompete other clonotypes and finally form a predominant population. This TCR identification method can be used to clone virus-specific TCRs efficiently from cancer patients and is easily adoptable by any laboratory.
\end{abstract}

Keywords: TCR cloning, Immunotherapy, Virus-specific TCRs, TCR transduced T cells

\section{Introduction}

Viral infections cause approximately $10-12 \%$ of all human malignancies worldwide. To date, at least 8 viruses have been associated with human cancers, including Epstein-Barr virus (EBV), papillomavirus (HPV), Kaposi's sarcoma-associated herpesvirus (KSHV), Merkel cell polyomavirus (MCV), hepatitis B virus (HBV), human T lymphotropic virus type-1 (HTLV-1), hepatitis $\mathrm{C}$ virus (HCV) and human immunodeficiency virus (HIV) [1]. In the life cycle of some viruses, especially EBV and HPV, virus-derived oncogenic proteins are always present in malignant cells, making them excellent targets for $\mathrm{T}$ cells. For example, the high-risk HPV-encoded oncoproteins E6 and E7 are essential for the generation and maintenance of HPV-related malignancies [2, 3]. All

\footnotetext{
*Correspondence: mingjunw@szinno.com

${ }^{\dagger}$ Lei Chen and Lianhua Dong contributed equally to this work.

${ }^{1}$ Department of Research and Development, Shenzhen Institute

for Innovation and Translational Medicine, Shenzhen International

Biological Valley-Life Science Industrial Park, Dapeng New District,

Shenzhen, China

Full list of author information is available at the end of the article
}

EBV-associated cancers express some EBV latency antigens (EBNA1, EBNA2, EBNA3-3C, EBNA-LP, LMP1 and LMP2) [4]. These intracellular or membrane proteins can be processed and presented on the host cell surface in the context of MHC molecules and recognized by $\mathrm{T}$ cell receptors (TCRs) as foreign antigens. Therefore, compared with traditional cancer therapies, adoptive T-cell therapies (ACTs) may have unique advantages in eradicating virus-associated malignancies $[5,6]$.

Over the past decades, ACT has achieved significant clinical efficacy in the treatment of many human malignancies [7-9]. It has also been demonstrated that the adoptive transfer of virus-specific cytotoxic $\mathrm{T}$ cells isolated and expended directly from tumor specimens (i.e., TILs) can mediate durable and even curative responses [10-12]. However, the widespread use of TILs has been limited by the challenge of consistently isolating autologous viral-specific TILs for each patient, a long timeframe to validate reactive TILs, limited in vivo proliferation potential of the final cell product, and a lack of standardized manufacturing processes [13-15]. In contrast, TCR gene-modified $\mathrm{T}$ cells can redirect nonspecific $\mathrm{T}$ cells 
against a predefined antigen and provide an attractive alternative to treat virus-related malignancies $[16,17]$.

The discovery of TCR was the key step in the development of TCR-T cell therapy. Several approaches have been explored to identify tumor-specific TCRs. The conventional approach involves $\mathrm{T}$ cell cloning through multiple rounds of limiting dilution to isolate reactive $\mathrm{T}$ cell single clones. This method is labor intensive and time consuming [18, 19]. Multimer- or activation markerguided cell isolation facilitates the discovery of antigen-specific $\mathrm{T}$ cells, but it requires the frequency of the antigen-specific $\mathrm{T}$ cells to be above the detection limit of flow cytometry [20, 21]. In recent years, several single-cell-based approaches have been developed [22-24]. These approaches involve the distribution of a single $\mathrm{T}$ cell into 96-well or 384-well plates through flow cell sorting or droplet microfluidics, RT-PCR to amplify paired TCR $\alpha$ and $\beta$ chains, and subsequent Sanger sequencing. Although these approaches are highly efficient, they are still expensive and not readily available in most labs.

Here, we present an efficient method to efficiently isolate virus-specific TCRs from virus-positive cancer patients. First, we found that many virus-positive patients have endogenous $\mathrm{T}$ cell responses against viral antigens, even though the immunogenicity of several viral antigens is low. Second, unlike the isolation of neoantigen-reactive TCRs, we only need one TCR with good properties against a predetermined viral epitope. We hypothesized that after elimination of most nonspecific $\mathrm{T}$ cells, a lead functional clone could outcompete other clones due to its strong proliferation potential. Therefore, we used a one-round nonstringent limiting dilution to isolate highly reactive polyclones and expanded these polyclones directly. Tetramer-positive cells were isolated from polyclones that could thrive and subjected to library preparation (5' RACE technique [25]) and next-generation sequencing (NGS). In many cases, we retrieved only one dominant full-length $V \alpha$ and $V \beta$ chain that could pair successfully. By using this approach, we quickly isolated TCRs against EBV, HPV and CMV. All TCRs showed high transduction efficiency, good alpha- and beta-chain pairing, high avidity and potent in vitro function.

\section{Results}

\section{An efficient method to isolate virus-specific TCR}

Peripheral blood mononuclear cells (PBMCs) collected from HLA-A2-positive patients with different malignancies, including nasopharyngeal carcinoma (NPC), metastatic cervical cancer and melanoma were used to isolate virus-specific TCRs. Compared with PBMCs from healthy donors, PBMCs from cancer patients showed higher endogenous reactivities to distinct viral antigens (Fig. 1 and Additional file 1: Fig.

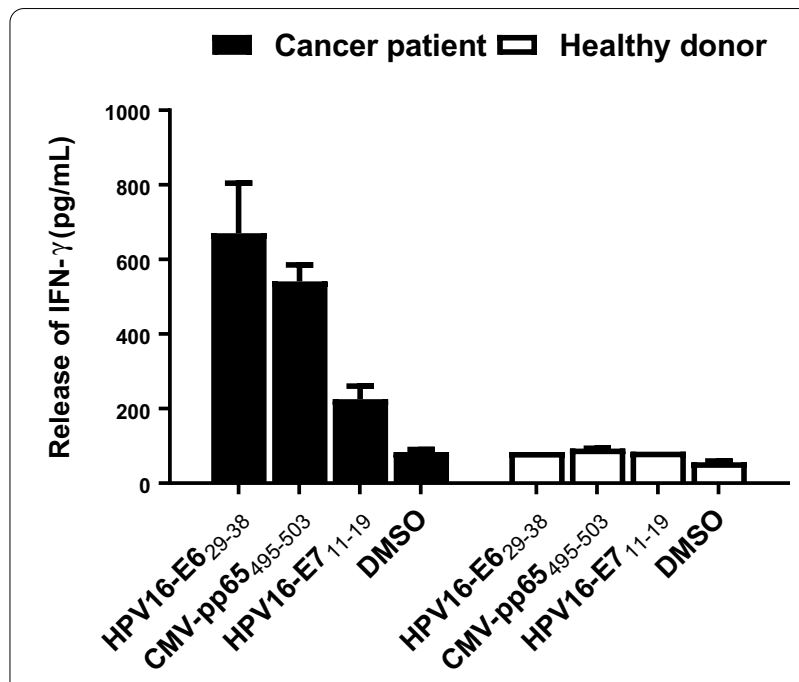

Fig. 1 High endogenous reactivities to viral antigens of PBMCs from cancer patients. PBMCs from a healthy donor and a patient with metastatic cervical cancer were cocultured with $\mathrm{T} 2$ cells pulsed with indicated peptides or DMSO for 16-18 h, respectively. The release of IFN- $\gamma$ in the cocultured supernatant were detected by ELISA. The IFN- $\gamma$ release of PBMCs from the cancer patient were three to eightfold higher than that of PBMCs from the healthy donor. Similar results were observed in patients with metastatic melanoma and NPC

S1), which indicates that (1) these epitopes are immunogenic and feasible targets for human T cells and (2) cancer patients would be a good source of virus-specific TCRs. As illustrated in Fig. 2, $\mathrm{CD}^{+} \mathrm{T}$ cells were first sensitized with irradiated, peptide-pulsed autologous PBMCs. Since the target epitopes are MHC-I restricted, we purified $\mathrm{CD}^{+} \mathrm{T}$ cells to eliminate the interference of a large number of nonspecific $\mathrm{CD} 4^{+} \mathrm{T}$ cells at the beginning. Then, cells from strong positive wells (release of IFN- $\gamma, \mathrm{OD}_{450} \geq 1$ after subtracting the control background signal) were mixed and subjected to a nonstringent limiting dilution (3 cells/well). We tested limiting dilutions with $0.3,3$ and 6 cells per well and found that when a stringent limiting dilution strategy was used ( 0.3 cells/well), most $T$ cells failed to grow to a sufficient number in 2 weeks. When cloning with 3 or 6 cells/well, most cells grew enough in 2 weeks for testing reactivity. Both dilution rates gave equivalent positive wells but higher background signal when using 6 cells/well. Polyclones from strongly positive wells were selected and expanded in T25 flasks. Antigen-specific $\mathrm{T}$ cells were further purified through tetramer selection of polyclones that grew vigorously. Finally, a full-length library was established, and TCR chains were retrieved by NGS. Interestingly, the NGS results showed one dominant TCR (Fig. 3). For example, the sequencing results of HPV16-E7-specific $\mathrm{T}$ 

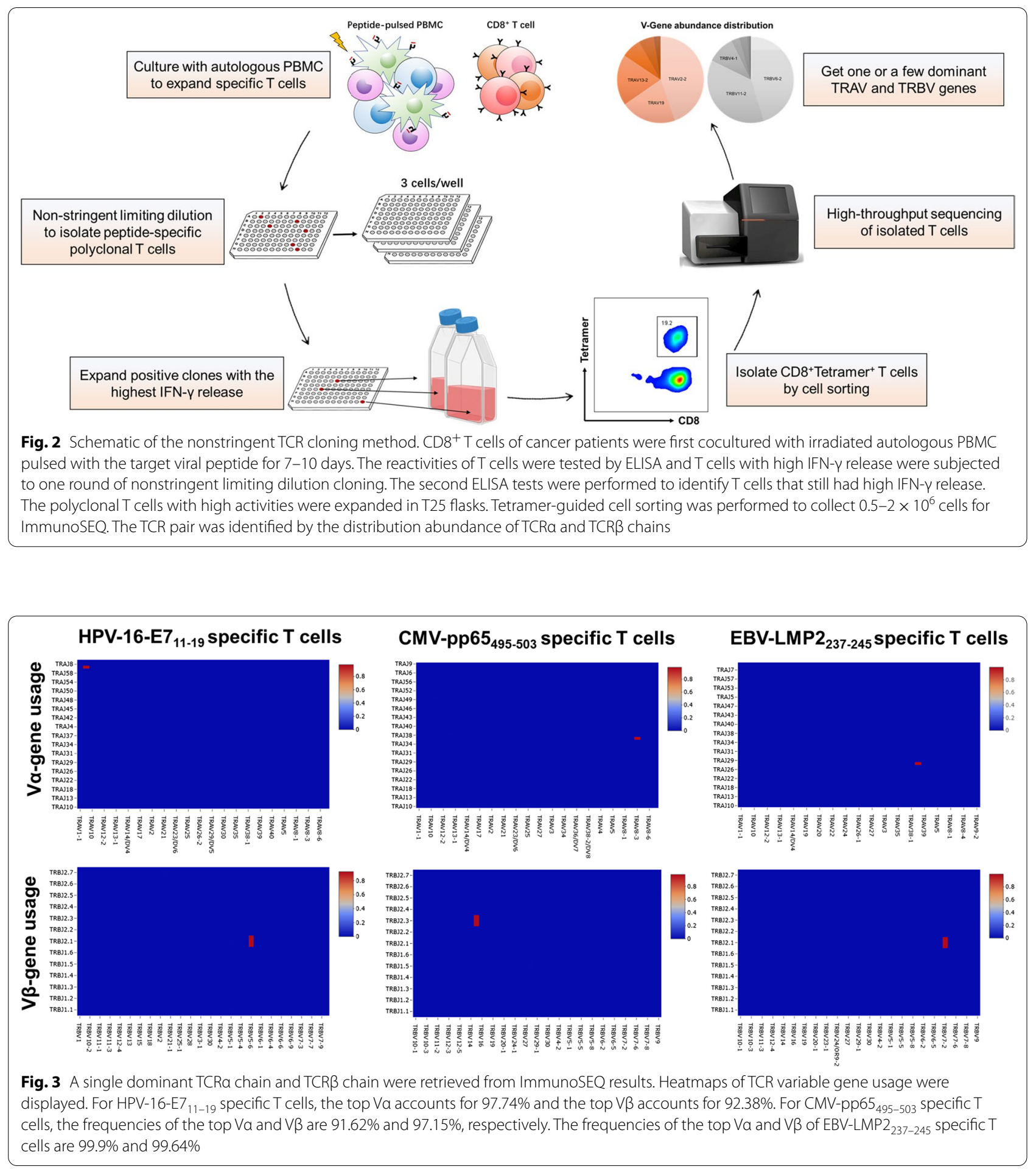

cells showed a single dominant TCR alpha chain (frequency: 97.74\%) and beta chain (frequency: 92.38\%), suggesting that the final $\mathrm{T}$ cells were monoclonal. We isolated 6 TCRs by this approach: G8 TCR targets to HPV16-E7 ${ }_{11-19}$, A6 TCR directs to CMVpp65 ${ }_{495-503}$,
A4 TCR recognizes $\mathrm{LMP}_{356-364}$, and A12, C5 and G1 recognize LMP2 $2_{426-434}$. All the viral epitopes are HLA$\mathrm{A}^{*}$ 02:01 restricted (Additional file 1: Fig. S2). 
TCRs generated by our approach can be successfully expressed and paired on transduced $\mathrm{T}$ cells

To investigate whether the TCRs isolated by our approach were functional, we cloned the DNA encoding target TCRs into an MSGV1 retroviral vector. The human TCR constant regions were replaced with their mouse counterparts. One concern about the safety of TCR-transduced $\mathrm{T}$ cell therapy is the potential mispairing of introduced TCRs with endogenous TCRs [26, 27]. Although several protein engineering methods could be used to enhance the pairing of introduced TCRs [28], it would be best to use a TCR with inherently good pairing capacity. All TCRs isolated by using our method showed high expression and good pairing with transduced $\mathrm{T}$ cells, as demonstrated by the equivalent percentages of tetramer ${ }^{+}$and mTRBC $^{+}$cell populations (Fig. 4a, b). The variable region sequences of the introduced TCRs were found to be the major determinant of the mispairing level [29]. Therefore, TCRs isolated by our method presumably have innately good characteristics.

\section{The selected TCRs specifically recognize viral epitopes} and have high functional avidity

Next, we tested the functional avidity of isolated TCRs. All TCR-T cells specifically recognized cognate peptideloaded T2 cells but not control cells (DMSO or irrelevant peptide-loaded T2 cells) (Fig. 5a-c). CMV and EBV TCR-transduced T cells (A6, A12, C5 and G1) also recognized epitopes from mutant antigens (CMVpp65 $495-503$ : NLVPIVATV, M499I and EBV-LMP2 ${ }_{356-364: \text { FLY- }}$ ALALLL, Y358C). All TCR-T cells showed a cytokine response (IFN- $\gamma$ ) in a dose-dependent manner and could respond to $\mathrm{T} 2$ cells pulsed with their cognate peptide at a concentration as low as $10 \mathrm{pM}$, indicating that all TCRs had high functional avidity (Fig. 6a-d).

\section{Virus-specific TCR-T cells specifically recognize and kill cancer cells expressing HLA-A2 and viral antigens}

We next performed experiments to confirm that TCR-T cells specifically recognize and kill human cancer cells expressing HLA-A2 and viral antigens. G8 TCR-T cells were cocultured with the human cervical cancer cell lines CaSki $\left(\mathrm{HLA}_{-} \mathrm{A}^{+}, \mathrm{E}^{+}{ }^{+}\right.$) or $\mathrm{SiHa}\left(\mathrm{HLA}-\mathrm{A} 2^{-}, \mathrm{E}^{+}\right) . \mathrm{G} 8$ TCR-T cells specifically reacted with CaSki but not $\mathrm{SiHa}$. The recognition of CaSki by G8 TCR-T cells was significantly inhibited by antibody blockade of HLA class ABC (antibody clone W6/32) but not HLA class BC molecules (antibody clone B1.23.2), which demonstrated that target recognition was HLA-A restricted (Fig. 7a). In the lactate dehydrogenase (LDH) cytotoxicity assay, both G8 TCRtransduced $\mathrm{CD} 4^{+}$and $\mathrm{CD} 8^{+} \mathrm{T}$ cells could lyse CaSki cells at a low effector-to-target ratio (Fig. 7b), indicating that the affinity of the G8 TCR was high; thus, the recognition of pMHC was $\mathrm{CD}^{+}$independent. Although the role of CMV in glioblastoma multiforme (GBM) has been controversial, some groups have reported the presence of CMVpp65 antigen in GBM tumors as a potential target for T cell therapy [30, 31]. Therefore, we constructed two CMVpp65-expressing glioblastoma cell lines (U87pp65-EGFP and T98-pp65-EGFP) to evaluate the in vitro function of A6 TCR-transduced T cells. A6 TCR-T cells specifically recognized CMVpp65-expressing cancer cells but not empty vector-transduced cancer cells (Fig. 7c). Similarly, A6 TCR-T cells killed U87-pp65-EGFP cells at a low effector-to-target ratio. The results showed that the selected virus-specific TCRs have potent in vitro functions (Fig. 7d).

\section{a}

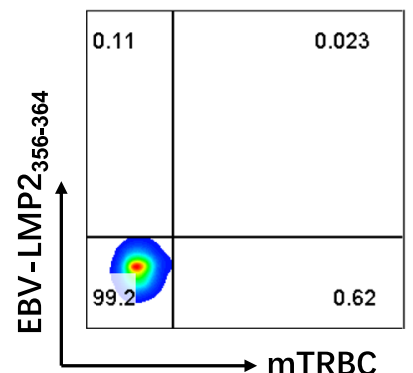

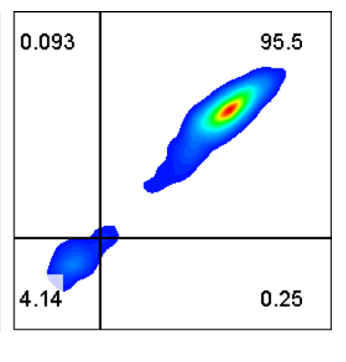

b

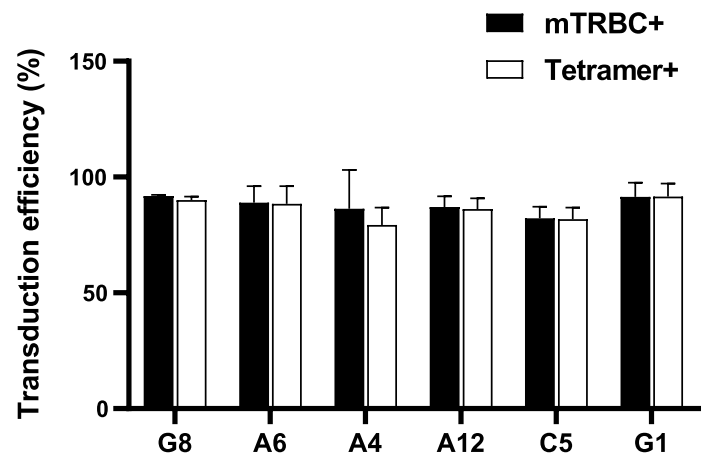

Fig. 4 TCRs that identified by our method are successfully expressed and paired on transduced T cells. a A representative result of flow cytometry analysis. Plotted cells were gated on lymphocytes (SSC $\times$ FSC), live cells $\left(7-\mathrm{AAD}^{-}\right)$, and $\mathrm{CD}^{+}{ }^{+}$cells. The percentage of tetramer ${ }^{+}$and $\mathrm{mTRBC}^{+}$ cells were calculated. $\mathbf{b}$ Transduction efficiency of TCRs calculated by tetramer and anti-mTRBC antibody staining. Data are representative of two independent experiments and values are expressed in mean \pm SEM 


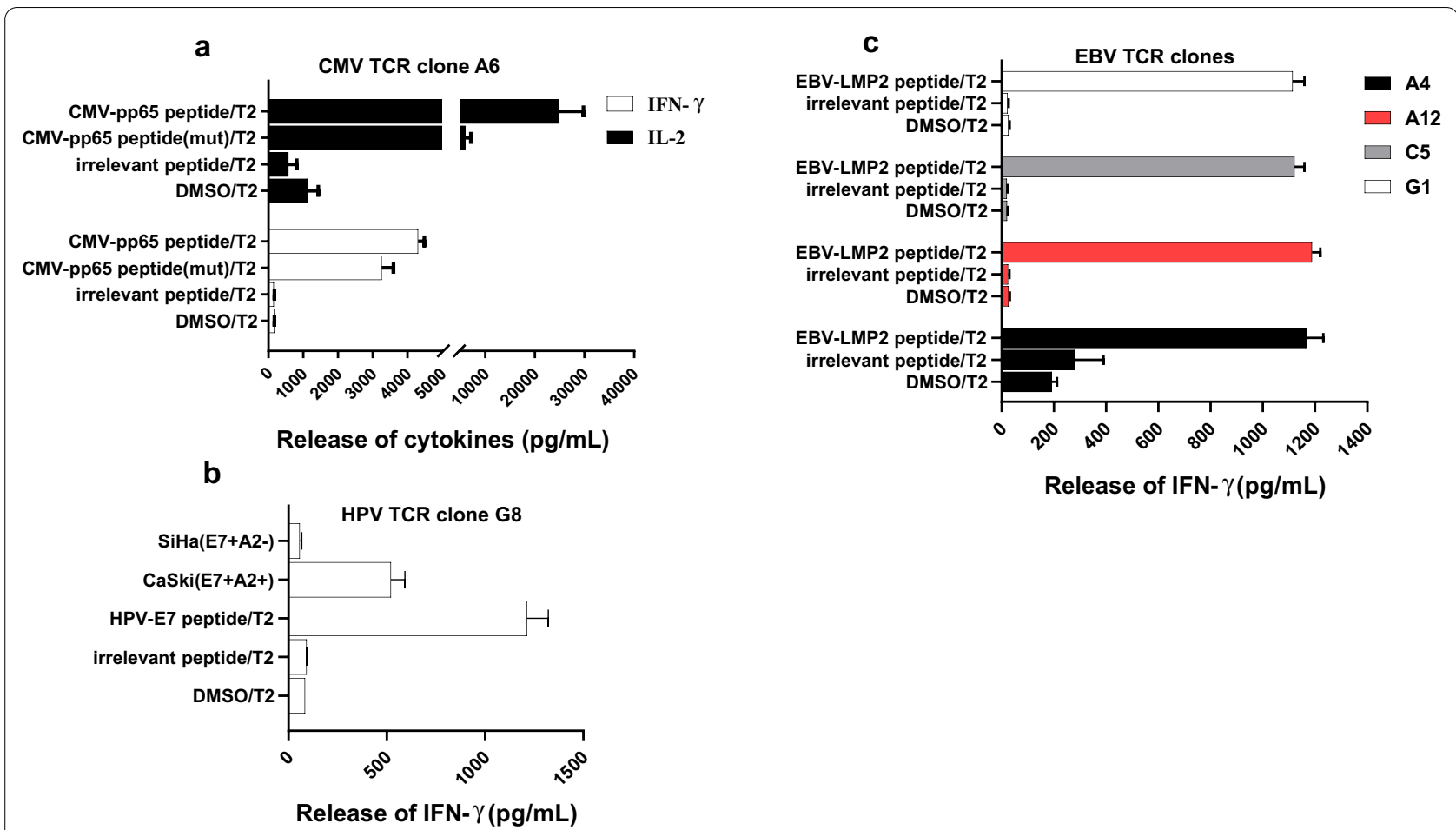

Fig. 5 TCR-transduced T cells specifically recognized cognate peptide-loaded T2 cells. CMV-specific TCR-T cells (a), EBV-specific TCR-T cells (b) and HPV-specific TCR-T cells (c) were cocultured with cognate peptide, irrelevant peptide or DMSO pulsed T2 cells. The release of IFN- $\gamma$ or IL-2 in the cocultured supernatant were detected by ELISA. Error bars represent the SEM for 3 technical replicates

HPV16-E7 specific TCR-T cells suppress the progression of HPV16-E7 ${ }^{+}$tumor in an immunodeficient mice model After confirming the cancer-killing ability of virus-specific TCR-T cells in vitro, we next tested the potential antitumor activity of TCR-T cells in NCG mice. Considering the availability of cancer cell lines expressing both target antigen and HLA, we chose cervical cancer as a model to evaluate the in vivo function of TCR-T cells. NCG mice implanted subcutaneously with CaSki-luc cells on day-21, were administered with phosphate-buffered saline (PBS), untransduced $\mathrm{T}$ cells (Ctrl-T) or G8 TCR-T cells (TCR-T) (Fig. 8a-c). A single intravenous injection of $4 \times 10^{6} \mathrm{G} \mathrm{TCR}^{+} \mathrm{T}$ cells was performed on day 0 with an intraperitoneal injection of IL-2 (50,000 U per day) on days 0-2. As shown in Fig. $8 \mathrm{~b}$, tumors continued to grow in the PBS group and Ctrl-T group, whereas the administration of G8 TCR-T cells resulted in significant suppression of the growth of CaSki-luc tumors. $(P<0.05)$. On day 27, all mice were sacrificed and the tumors were excised (Fig. 8c). Consistent with the bioluminescence imaging result, we found a marked reduction in tumor sizes of all mice in the TCR-T group compared with those in the PBS groups and the Ctrl-T group. All these results demonstrated that G8 TCR-T cells at a medium dose of
$4 \times 10^{6} \mathrm{TCR}^{+}$cells were able to significantly delay the progression of CaSki-luc tumors.

\section{Materials and methods}

\section{Cell lines and culture media}

CaSki, SiHa, U87, T98G and T2 cell lines were purchased from ATCC. Cell lines were cultured in RPMI1640 (CaSki, SiHa and T2), or DMEM (293GP, U87 and T98G) supplemented with 10\% (v/v) FBS (Lonsera), GlutaMAX (Life Technologies), $100 \mathrm{U} / \mathrm{ml}$ penicillin (Life Technologies) and $100 \mu \mathrm{g} / \mathrm{ml}$ streptomycin (Life Technologies). U87-pp65-EGFP, and T98-pp65-EGFP are U87 or T98-based cell lines with stable expression of pp65-GFP. These cell lines were generated by transduction of retroviral vectors encoding target proteins, and a subsequent cell sorting of $\mathrm{GFP}^{+}$cells with flow cytometry. All cell lines that used in the experiments tested negative for mycoplasma. The culture medium for $\mathrm{T}$ cell in vitro peptide sensitization was X-VIVO ${ }^{\mathrm{TM}}$ 15 (Lonza) with 5\% (v/v) human AB serum and $50 \mathrm{U} / \mathrm{ml}$ IL-2 (Peprotech). The medium used for quick expansion of $\mathrm{T}$ cell was X-VIVO ${ }^{\mathrm{TM}} 15$ with $10 \%(\mathrm{v} / \mathrm{v})$ FBS serum and $300 \mathrm{U} / \mathrm{ml} \mathrm{IL-2}$ and $50 \mathrm{ng} / \mathrm{mL}$ IL-15 (Peprotech). 

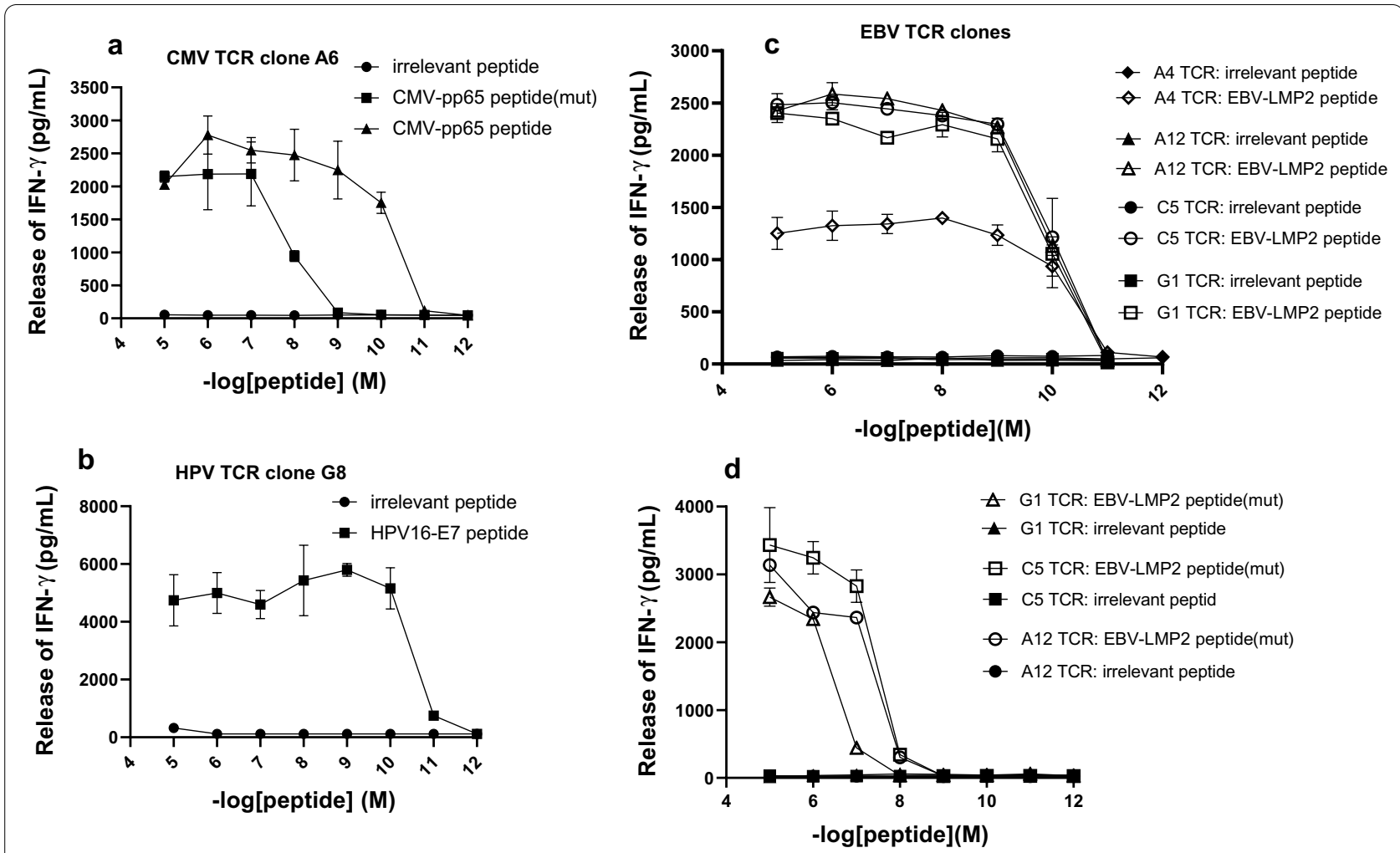

Fig. 6 Functional avidity assay of TCR-transduced T cells. CMV-specific TCR-T cells (a), HPV-specific TCR-T cells (b) and EBV-specific TCR-T cells (c) d) were cocultured with $\mathrm{T} 2$ cells that pulsed with peptides at the concentration indicated on the $x$-axis. The release of IFN- $\gamma$ in the cocultured supernatant were detected by ELISA. Error bars represent the SEM for 3 technical replicates

\section{Generation of virus-specific T cell clones}

PBMC were obtained by leukapheresis, prepared with density gradient centrifugation (human $\mathrm{T}$ lymphocyte separation medium from Dakewe Biotech), and cryopreserved. For in vitro sensitization, $\mathrm{CD}^{+} \mathrm{T}$ cells were first isolated from human PBMC using Dynabeads ${ }^{\mathrm{TM}} \mathrm{CD} 8$ positive isolation kit (Life Technologies), and then $3 \times 10^{5}$ $\mathrm{CD}^{+} \mathrm{T}$ cells were stimulated with irradiated, viral peptide-loaded autologous PBMC $\left(1 \times 10^{6}, \mathrm{CD}^{+} \mathrm{T}\right.$ celldepleted PBMC) in each well of a 24-well plate. Cultures were maintained individually for 7-10 days and replaced with fresh medium every 2 days. The reactivities of cells were tested by the IFN- $\gamma$ release assay against T2 cells pulsed with cognate viral peptides or irrelevant peptides. $T$ cell cultures with pronounced and specific recognition of the target epitope $\left(\mathrm{OD}_{450} \geq 1\right.$ after subtracting control signal) were subjected to the limiting dilution cloning (3 cells/well). Specifically, T cells were stimulated with $2 \times 10^{6}$ irradiated allogenic PBMC from three healthy donors and $30 \mathrm{ng} / \mathrm{mL}$ anti-CD3 antibody (OKT3) in the quick expansion medium. After 12-14 days of culture, plates were screened by ELISA again to isolate virus-specific $\mathrm{T}$ cells with high positive signal. The quick expansion protocol was used to expand positive T cells in T25 flasks [32]. After tetramer-guided selection, 1-2 $\times 10^{6} \mathrm{~T}$ cells were used to isolate RNA (RNeasy plus universal mini kit, Qiagen) and were sent to GENEWIZ (Nanjing, China) for ImmunoSEQ.

\section{Construction of TCR retroviral vector and T cell transduction}

TCR nucleotide sequences were synthesized and cloned into the MSGV1-1D3-28Z.1-3 mut (addgene) retroviral vector. The human TCR constant regions were replaced with murine TCR constant regions, and a furin P2A linker was used to connect the TCR chains in the $\alpha-\beta$ order. Retroviral vectors encoding the target TCRs were first generated by transfecting 293GP packaging cells with pMSGV1-TCR and VSV-G plasmids. Viral vector-containing supernatant were harvested 48 and $72 \mathrm{~h}$ post-transfection. PG13-TCR producer cells were established by two consecutive transductions of PG13 cells with harvested viral supernatant. Human PBMC from healthy donors were isolated as mentioned above. $\mathrm{CD}^{+}$ $\mathrm{T}$ cells were isolated from PBMCs with Dynabeads ${ }^{\mathrm{TM}}$ untouched $^{\mathrm{TM}}$ human $\mathrm{T}$ cells kit (Life Technologies) and stimulated for 2 days with Dynabeads human T-activator CD3/CD28 (Life Technologies) at a bead to cell ratio of 


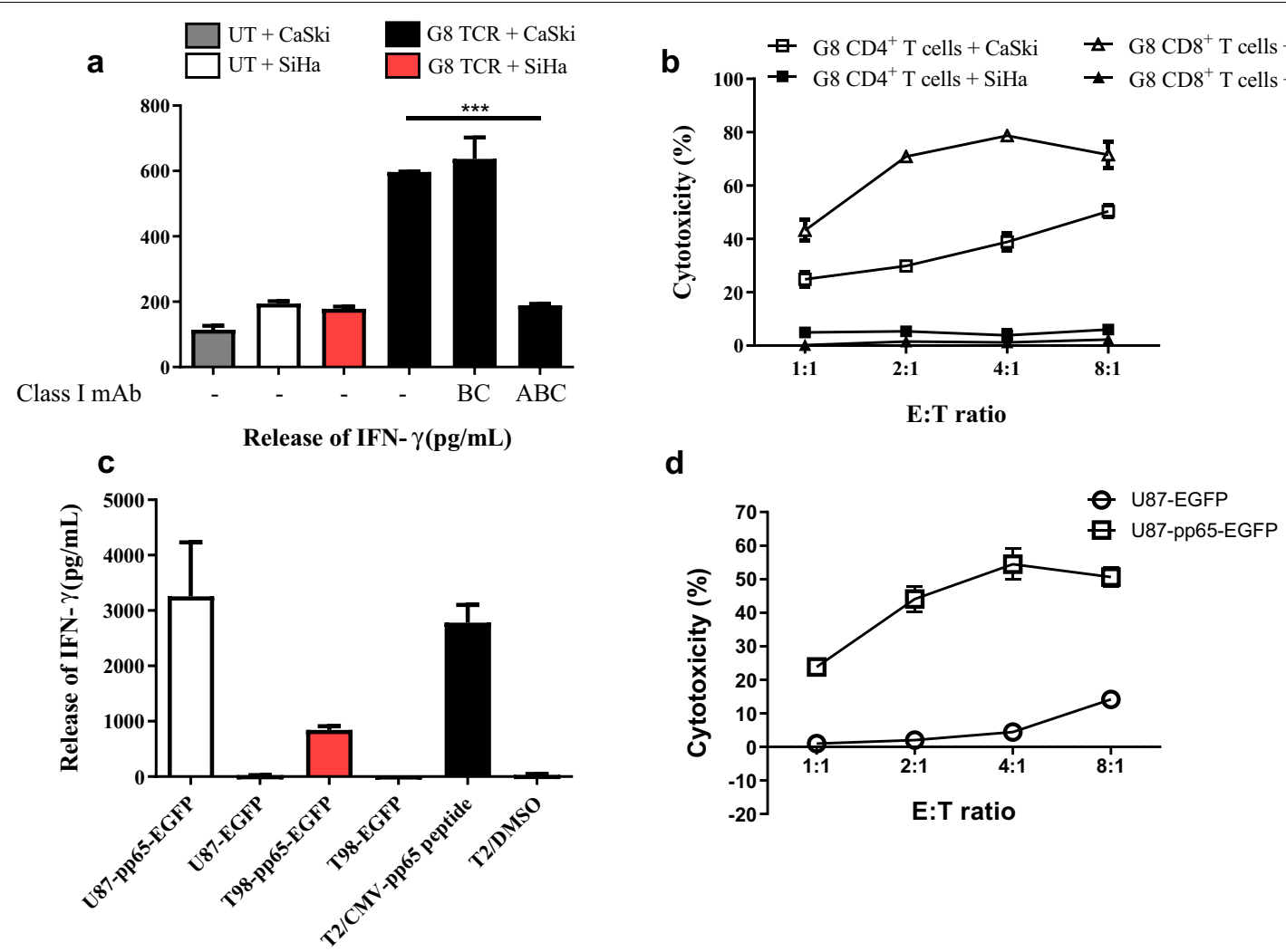

Fig. 7 TCR-transduced T cells recognize and kill cancer cells expressing target viral antigens in vitro. a MHC competition assay in which the effector

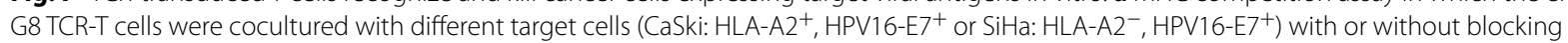
antibodies as indicated in $x$-axis. UT: untransduced T cells served as a negative control. $\mathbf{b} \mathrm{LDH}$ release assay of G8 TCR transduced CD4 ${ }^{+}$and $\mathrm{CD} 8^{+}$ T cells. c A6 TCR-T cells were cocultured with glioblastoma cell lines (U87 or T98) expressing CMV antigen or empty vector. $\mathbf{d}$ LDH release assay of A6 TCR-transduced T cells against U87 expressing CMV-pp65. The ratio of effector cells to target cells was indicated in the $x$-axis (E: T ratio). The percentage of cytotoxicity was calculated according to the manufacturers' instructions

1:1. Retroviral vectors that produced from PG13-TCR cells were preloaded into RetroNectin-coated 6-well plates (Takara), and activated $\mathrm{T}$ cells were transduced with two cycles of spinoculation.

\section{Flow cytometry}

The following fluorescently conjugated antibodies were purchased from BD Biosciences: CD3-BV605/APC/PE (SK7), CD8-APC-H7/FITC (HIT8a), CD4-PE/BV786/ APC (SK3), murine TCR $\beta$ constant region (mTRBC)PE-Cy7. Tetramers (HLA-A*02:01-HPV16-E7 ${ }_{11-19}$, HLA-A*02:01-EBV-LMP2 ${ }_{356-364,}$ HLA-A*02:01-EBVLMP2 $_{426-434}$ and HLA-A*02:01-CMVpp65 $495-503$ ) were purchased from MBL International. Data were acquired with a BD FACSAria ${ }^{\mathrm{TM}}$ II flow cytometer (BD Biosciences) and analyzed with FlowJo software (FlowJo).

\section{T cell in vitro functional assays}

For cytokine production assays, $1 \times 10^{5} \mathrm{~T}$ cells were cocultured with $5 \times 10^{4}$ target cells in 96-well U-bottom plates at $37{ }^{\circ} \mathrm{C}$. For peptide titration experiments, T2 cells were pulsed with different concentrations of peptides overnight. After washing, $5 \times 10^{4}$ peptide-pulsed $\mathrm{T} 2$ cells were cocultured with $1 \times 10^{5} \mathrm{~T}$ cells as mentioned above. Coculture supernatants were harvested after $16-18 \mathrm{~h}$, and IFN- $\gamma$ concentration was measured by ELISA (Thermo Scientific). Cytotoxicity of $\mathrm{T}$ cells was determined by lactate dehydrogenase (LDH) release assays. $\mathrm{T}$ cells were cocultured with target cells at the indicated effector-to-target ratios, and LDH in coculture media were quantified according to the manufacturers' instructions (Pierce LDH cytotoxicity assay kit, Thermo Scientific).

\section{Treatment of cervical cancer in a xenograft murine model} Animal research protocols were approved by the Animal Experiment Ethics Committee of Shenzhen Second People's Hospital, Shenzhen, Guangdong Province, China. Female (4-6 weeks old) NCG mice (NOD-Prkdc ${ }^{e m 26} I l$ $\left.2 g^{e m 26} / \mathrm{Nju}\right)$ were purchased from GemPharmatech 


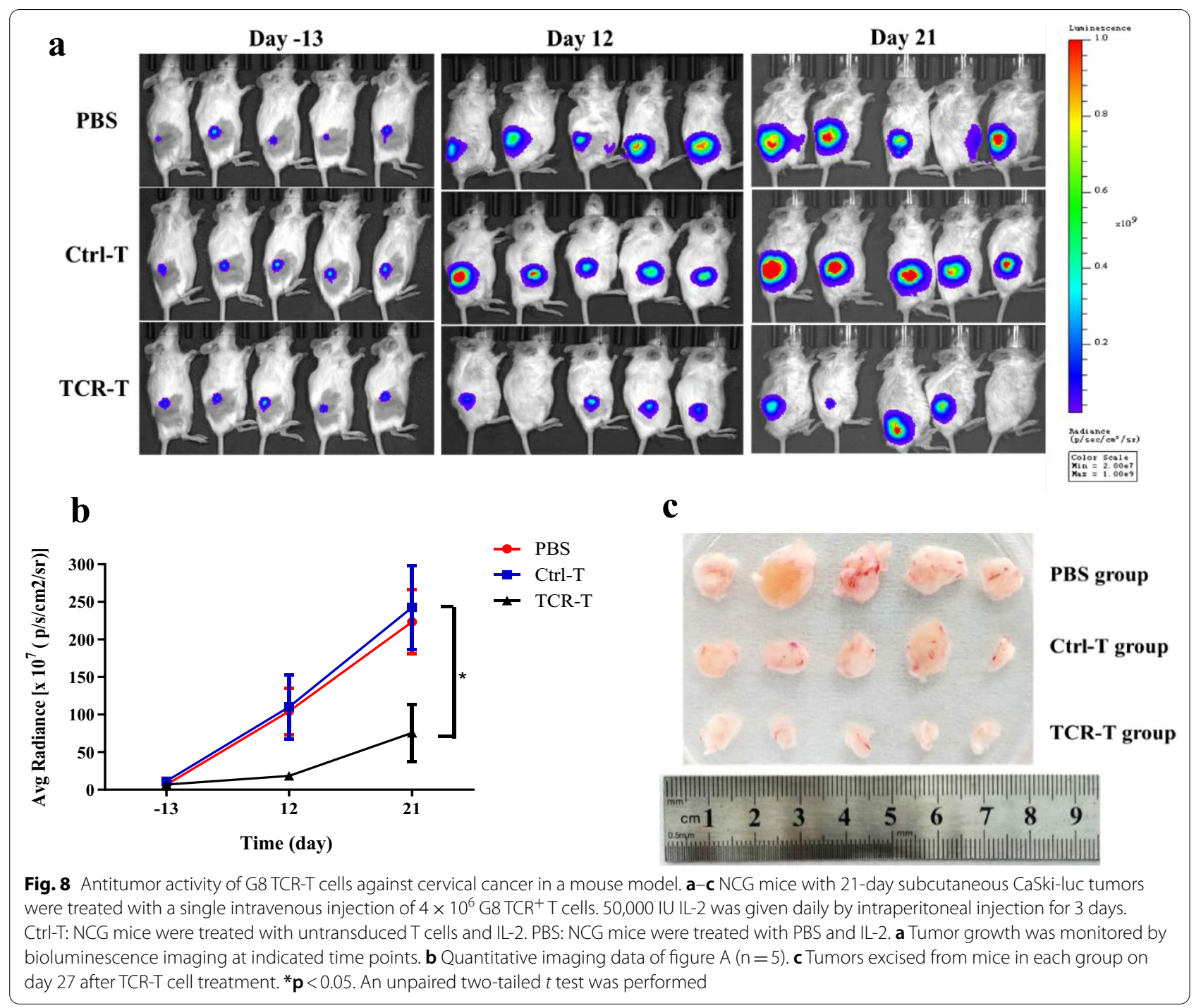

(Jiangsu, China). Tumors were initiated by a subcutaneous injection of $2 \times 10^{6}$ CaSki-luc cancer cells on the flank of mice. Twenty-one-days following tumor initiation, $\mathrm{T}$ cells (E7 specific TCR-T cells or untransduced T cells were administered by tail vein injection. Mice received adjuvant IL-2 (Jiangsu Kingsley Pharmaceutical Co., Ltd.) $50,000 \mathrm{IU}$ by intraperitoneal injection daily for 3 days beginning immediately after $\mathrm{T}$ cell infusion. Tumor load was measured by bioluminescence imaging. Isofluraneanesthetized animals were imaged using the IVIS system (Xenogen Corp.) $10 \mathrm{~min}$ after intraperitoneal injection of $150 \mathrm{mg} / \mathrm{kg}$ VivoGlo ${ }^{\mathrm{TM}}$ luciferin (Promega). Mice were regularly examined for weight loss, or signs of stress, and euthanized by cervical dislocation according to the preset criteria. At the end of the experiment, Heparinized blood samples were collected by intracardiac puncture under general anesthesia (a single dose of intraperitoneal injection of $250 \mathrm{mg} / \mathrm{kg}$ ketamine and $10 \mathrm{mg} / \mathrm{kg}$ xylazine). After that, mice were immediately euthanized by cervical dislocation, and tumors and tissues were collected.

\section{Statistics}

Statistical analyses were performed with Prism 5 (GraphPad Software). A two-tailed unpaired $t$ test was used to compare the differences between G8 TCR-T cell group and control groups. A $P$ value of less than 0.05 was considered significant.

\section{Conclusions}

TCR discovery has played a key role in the development of effective TCR-T cell therapy. The highly diverse TCR repertoire in the human body makes isolation of rare tumor-specific $T$ cells challenging. Incorrect pairing of TCR $\alpha$ and TCR $\beta$ chains will lead to the loss 
of function or specificity; thus, most $\mathrm{T}$ cell cloning approaches involve a $\mathrm{T}$ cell singularization process. The conventional singularization process involves iterative rounds of stringent limiting dilution and functional tests, whereas most recent methods adopted functionindependent singularization, such as multimer-guided single-cell sorting, RT-PCR and subsequent abundant TCR validation. We found that cancer patients are a good source of cloned virus-specific $\mathrm{T}$ cells, and stringent singularization was not necessary. $\mathrm{T}$ cell clones with strong proliferation potential could outcompete other cells and finally dominate the whole population. Therefore, we combined several nonstringent methods to narrow the repertoire of target $\mathrm{T}$ cells in a stepwise manner and finally obtained a single TCR with good properties.

The benefit of isolating TCR from cancer patients has been demonstrated [33]. Patients who had complete responses to immunotherapies, including immune checkpoint blockage [34], vaccines [35], and adoptive $\mathrm{T}$ cell transfer [36, 37] would be the best candidates to isolate highly functional TCRs. Although we could not test the expression of viral antigens in these patients and their treatment results are unknown, the PBMCs of these patients showed a higher endogenous $\mathrm{T}$ cell response to distinct viruses than normal donors. This is reasonable because HPV and EBV are direct causes of cervical cancer and NPC, respectively, while nearly all people have CMV infection, which may reactivate in an immunesuppressive environment. Due to a relatively high initial frequency of virus-specific $T$ cells in cancer patients, one round of in vitro peptide stimulation is usually sufficient for ELISA, which shortens the timeframe.

Our approach also saves time and has a higher success rate in the cloning process. Considering the statistical nature of the limiting dilution, a stringent dilution rate $(0.3-0.5 \mathrm{cell} /$ well) was usually used to guarantee that the final colonies arise from single cells. However, 6-10 times more plates and labor are required to screen the same amount of $\mathrm{T}$ cells as using our method. Moreover, because of T-cell exhaustion, some antigen-specific $T$ cells may gradually lose their function or fail to grow in the process of repeated expansion. We found that 3 cells/ well consistently gave high positive clones with low background signal. With the increase in cell number per well, the background signal increased as well.

Tetramer-guided cell sorting facilitates the isolation of antigen-specific $\mathrm{T}$ cells. However, it requires the frequency of positive cells to be beyond the detection limit of flow cytometry $(0.1-1 \%)$, and strong tetramer staining is not always correlated with good function. Therefore, we used it in the last polishing step to further purify $\mathrm{T}$ cells and retrieve function-exhausted clones.
NGS of bulk T cells has been used to retrieve tumorspecific TCRs based on the abundance of $V \alpha$ and $V \beta$ [38]. Due to the variation in library construction and PCR with multiple $V \alpha$ and $V \beta$ primers, the top $V \beta$ may not correctly pair with the top $V \alpha$. In addition, it has been reported that up to $30 \%$ of $\mathrm{T}$ cells express two distinct $\alpha$ chains [39]. To reduce the variation in NGS, we constructed a full-length library with 5 ' RACE. In most cases, we obtained only one dominant $\mathrm{V} \alpha$ and $\mathrm{V} \beta$ chain (frequency $>90 \%$ ), and the top V $\beta$ was always correctly paired with the top $V \alpha$.

In summary, the modified TCR identification method proposed in this paper not only reduces the time and labor compared with conventional limiting-dilutionbased $\mathrm{T}$ cell cloning but is also more cost effective than single-cell-based techniques. Our method is readily adoptable to any labs equipped with conventional instruments and can be integrated into an automated process as well. Furthermore, this approach allows $\mathrm{T}$ cell clones with potent function and proliferation properties to automatically appear, omitting the tedious validation phase.

\section{Abbreviations}

TCR: T cell receptor; EBV: Epstein-Barr virus; HPV: Papillomavirus; KSHV: Kaposi's sarcoma-associated herpesvirus; MCV: Merkel cell polyomavirus; HBV: Hepatitis B virus; HTLV-1: Human T lymphotropic virus type-1; HCV: Hepatitis C virus; HIV: Human immunodeficiency virus; ACT: Adoptive T-cell therapies; TILs:Tumorinfiltrating lymphocytes; PBMCs: Peripheral blood mononuclear cells; GBM: Glioblastoma multiforme.

\section{Supplementary Information}

The online version contains supplementary material available at https://doi. org/10.1186/s12865-021-00455-3.

Additional file 1. Fig. S1. The high percentage of virus-specific T cells in the PBMC of cancer patients. (a) PBMCs from a healthy donor and a patient with metastatic melanoma were cocultured with T2 cells pulsed with indicated peptides or DMSO for 16-18 hours, respectively. The release of IFN- $\gamma$ in the cocultured supernatant was detected by ELISA. (b) PBMC from a healthy donor and a patient with NPC were stained with EBV-LMP2 tetramer, and the cells were gated on tetramer-positive cells (SSC $\times$ PE). Fig. S2. The CDR3 sequences of isolated virus-specific T cell clones.

\section{Acknowledgements \\ Not applicable.}

\section{Authors' contributions}

LC, LHD, GT and MJW designed the study and planned the experiments. LC and LHD performed the experiments. LC and LHD analyzed the data. YPM, JTW and DJQ performed the PBMC preparation and cell line construction. LC and MJW wrote the manuscript. LC, LHD, YPM, JTW, DJQ, GT and MJW edited the manuscript.

\section{Funding}

This research was supported by Shenzhen Peacock Plan (Grant Number, KQTD20130416114522736); Shenzhen Basic Research Program (Grant Numbers, JCYJ20180507182902330 and JCYJ20170412102821202), Special Funds for Dapeng New District Industry Development (Grant Numbers, KY20180214, KY20180105, KY20170203, and PT201901-12). 


\section{Availability of data and materials}

All data generated or analysed during this study are included in this published article.

\section{Declarations}

Ethics approval and consent to participate

Animal research protocols were approved by the Animal Experiment Ethics Committee of Shenzhen Second People's Hospital, Shenzhen, Guangdong Province, China. All methods involving animals use were carried out in compliance with the ARRIVE guidelines and approved by the Animal Experiment Ethics Committee of Shenzhen Second People's Hospital.

\section{Consent for publication}

Not applicable.

\section{Competing interests}

No potential conflict of interest reported by the authors.

\section{Author details}

'Department of Research and Development, Shenzhen Institute for Innovation and Translational Medicine, Shenzhen International Biological Valley-Life Science Industrial Park, Dapeng New District, Shenzhen, China. ${ }^{2}$ Department of Oncology, Shenzhen Second People's Hospital, The First Affiliated Hospital of Shenzhen University, Shenzhen 518035, China.

Received: 24 May 2021 Accepted: 14 September 2021

Published online: 28 September 2021

\section{References}

1. Tashiro H, Brenner MK. Immunotherapy against cancer-related viruses. Cell Res. 2017;27:59-73.

2. Tindle RW. Immune evasion in human papillomavirus-associated cervical cancer. Nat Rev Cancer. 2002;2:59-64.

3. Münger K, et al. Mechanisms of human papillomavirus-induced oncogenesis. J Virol. 2004;78:11451-60.

4. Taylor GS, Long HM, Brooks JM, Rickinson AB, Hislop AD. The immunology of Epstein-Barr virus-induced disease. Annu Rev Immunol. 2015;33:787-821.

5. Zsiros E, Tsuji T, Odunsi K. Adoptive T-cell therapy is a promising salvage approach for advanced or recurrent metastatic cervical cancer. J Clin Oncol. 2015;33:1521-3.

6. Lutzky VP, et al. Cytotoxic T cell adoptive immunotherapy as a treatment for nasopharyngeal carcinoma. Clin Vaccine Immunol. 2014;21:256-9.

7. Rosenberg SA, et al. Use of tumor-infiltrating lymphocytes and interleukin-2 in the immunotherapy of patients with metastatic melanoma. $\mathrm{N}$ Engl J Med. 1988;319:1676-80.

8. Dudley ME, et al. Adoptive cell transfer therapy following non-myeloablative but lymphodepleting chemotherapy for the treatment of patients with refractory metastatic melanoma. J Clin Oncol. 2005;23:2346-57.

9. Rosenberg SA, Restifo NP, Yang JC, Morgan RA, Dudley ME. Adoptive cell transfer: a clinical path to effective cancer immunotherapy. Nat Rev Cancer. 2008:8:299-308.

10. Stevanović S, et al. Complete regression of metastatic cervical cancer after treatment with human papillomavirus-targeted tumor-infiltrating $T$ cells. J Clin Oncol. 2015;33:1543.

11. Louis CU, et al. Adoptive transfer of EBV-specific T cells results in sustained clinical responses in patients with locoregional nasopharyngeal carcinoma. J Immunother. 2010;33:983-90.

12. Bollard CM, et al. Sustained complete responses in patients with lymphoma receiving autologous cytotoxic T lymphocytes targeting EpsteinBarr virus latent membrane proteins. J Clin Oncol. 2014;32:798-808.

13. Schmitt TM, Ragnarsson GB, Greenberg PD. T cell receptor gene therapy for cancer. Hum Gene Ther. 2009;20:1240-8.

14. Dudley ME, Wunderlich JR, Shelton TE, Even J, Rosenberg SA. Generation of tumor-infiltrating lymphocyte cultures for use in adoptive transfer therapy for melanoma patients. J Immunother. 2003;26:332-42.
15. Tran $K Q$, et al. Minimally cultured tumor-infiltrating lymphocytes display optimal characteristics for adoptive cell therapy. J Immunother. 2008;31:742-51.

16. Draper $L M$, et al. Targeting of HPV-16+ epithelial cancer cells by TCR gene engineered T cells directed against E6. Clin Cancer Res. 2015;21:4431-9.

17. Schumacher TN. T-cell-receptor gene therapy. Nat Rev Immunol. 2002;2:512-9.

18. Baker PE, GíLLíS S, Smith KA. Monoclonal cytolytic T-cell lines. J Exp Med. 1979:149:273-8.

19. Mariotti, S. \& Nisini, R. in T Cell Protocols. Methods in Molecular Biology ${ }^{T M}$ Vol. 514 (eds De Libero G.) 65-93 (Springer, 2009).

20. Bernhard, H., Schmidt, B., Busch, D. H. \& Peschel, C. in Adoptive Immunotherapy: Methods and Protocols, Methods Molecular Medicine Vol. 109 (eds Hoffmann MW Ludewig B) 175-184 (Springer, 2006).

21. Dössinger $\mathrm{G}$, et al. MHC multimer-guided and cell culture-independent isolation of functional T cell receptors from single cells facilitates TCR identification for immunotherapy. PLoS ONE. 2013;8:e61384.

22. Hu Z, et al. A cloning and expression system to probe T-cell receptor specificity and assess functional avidity to neoantigens. Blood. 2018;132:1911-21.

23. Segaliny Al, et al. Functional TCR T cell screening using single-cell droplet microfluidics. Lab Chip. 2018;18:3733-49.

24. Xi-zhi JG, et al. Rapid cloning, expression, and functional characterization of paired $a \beta$ and $\gamma \delta$ T-cell receptor chains from single-cell analysis. Mol Ther Methods Clin Dev. 2016;3:15054.

25. Zhu Y, Machleder E, Chenchik A, Li R, Siebert P. Reverse transcriptase template switching: a SMART ${ }^{\mathrm{TM}}$ approach for full-length CDNA library construction. Biotechniques. 2001:30:892-7.

26. Sebestyén $Z$, et al. Human TCR that incorporate $C D 3 \zeta$ induce highly preferred pairing between TCRa and $\beta$ chains following gene transfer. J Immunol. 2008;180:7736-46.

27. Govers C, Sebestyén Z, Coccoris M, Willemsen RA, Debets R. T cell receptor gene therapy: strategies for optimizing transgenic TCR pairing. Trends Mol Med. 2010;16:77-87.

28. Jin BY, et al. Engineered T cells targeting E7 mediate regression of human papillomavirus cancers in a murine model. JCI Insight. 2018;3:e99488.

29. Thomas S, et al. Targeting the Wilms tumor antigen 1 by TCR gene transfer: TCR variants improve tetramer binding but not the function of gene modified human T cells. J Immunol. 2007:179:5803-10.

30. Lucas KG, Bao L, Bruggeman R, Dunham K, Specht C. The detection of CMV pp65 and IE1 in glioblastoma multiforme. J Neurooncol. 2011;103:231-8.

31. Nair SK, et al. Recognition and killing of autologous, primary glioblastoma tumor cells by human cytomegalovirus pp65-specific cytotoxic T cells. Clin Cancer Res. 2014;20:2684-94.

32. Watanabe K, Goodrich J, Li C, Agha M, Greenberg P. Restoration of viral immunity in immunodeficient humans by the adoptive transfer of $T$ cell clones. Science. 1992;257:238-41.

33. Inderberg EM, Wälchli S. Long-term surviving cancer patients as a source of therapeutic TCR. Cancer Immunol Immunother. 2020;69:859-65.

34. Kamphorst $\mathrm{AO}$, et al. Proliferation of $\mathrm{PD}-1^{+} \mathrm{CD} 8 \mathrm{~T}$ cells in peripheral blood after PD-1-targeted therapy in lung cancer patients. Proc Natl Acad Sci U S A. 2017;114:4993-8.

35. Plon $\mathrm{SE}$, et al. Cytomegalovirus immunity after vaccination with autologous glioblastoma lysate. N Engl J Med. 2008;358:539-41.

36. Zhou J, Dudley ME, Rosenberg SA, Robbins PF. Persistence of multiple tumor-specific T-cell clones is associated with complete tumor regression in a melanoma patient receiving adoptive cell transfer therapy. J Immunother. 2005;28:53.

37. Marcinkowski B, et al. Cancer targeting by TCR gene-engineered T cells directed against Kita-Kyushu Lung Cancer Antigen-1. J Immunother Cancer. 2019:7:1-9

38. Howie $B$, et al. High-throughput pairing of $T$ cell receptor $a$ and $\beta$ sequences. Sci Transl Med. 2015;7:301ra131.

39. Han A, Glanville J, Hansmann L, Davis MM. Linking T-cell receptor sequence to functional phenotype at the single-cell level. Nat Biotechnol. 2014;32:684-92.

\section{Publisher's Note}

Springer Nature remains neutral with regard to jurisdictional claims in published maps and institutional affiliations. 